\title{
Téoros
}

Revue de recherche en tourisme

\section{Le Quartier international}

Un tonifiant pour le tourisme à Montréal

\section{Clément Demers, Bernard Lamothe et Louis-François Monet}

Volume 19, numéro 3, automne 2000

Organisations touristiques en mutation

URI : https://id.erudit.org/iderudit/1071740ar

DOI : https://doi.org/10.7202/1071740ar

Aller au sommaire du numéro

Éditeur(s)

Université du Québec à Montréal

ISSN

0712-8657 (imprimé)

1923-2705 (numérique)

Découvrir la revue

Citer cet article

Demers, C., Lamothe, B. \& Monet, L.-F. (2000). Le Quartier international : un tonifiant pour le tourisme à Montréal. Téoros, 19(3), 32-35.

https://doi.org/10.7202/1071740ar d'utilisation que vous pouvez consulter en ligne.

https://apropos.erudit.org/fr/usagers/politique-dutilisation/ 


\section{Le Quartier international}

\section{UN TONIFIANT POUR LE TOURISME À MONTRÉAL}

\section{Clément Demers, Bernard Lamothe et Louis-François Monet}

Le Quartier international de Montréal se définit comme un "projet d'aménagement urbain » qui permettra, notamment, de retisser les liens entre le Vieux-Montréal et le Centre des affaires, de susciter l'implantation d'organisations internationales à Montréal, de favoriser la réalisation de projets immobiliers de prestige et d'augmenter les retombées économiques liées à l'agrandissement du Palais des Congrès.

Ce court article ne mettra en relief que deux aspects de ce grand projet: le rôle des divers partenaires engagés dans sa réalisation et ses retombées sur le plan touristique.

\section{LE PRINCIPE DU PARTENARIAT}

Le projet du Quartier international de Montréal regroupe des partenaires des secteurs public, parapublic, institutionnel et privé. Il faut rappeler que ce type de partenariat, qui met à profit la complémentarité des ressources et des intérêts, n'est pas nouveau à Montréal et qu'on le trouve, sous diverses formes, aussi bien dans de grands projets - comme le Centre de commerce mondial ou la Cité du multimédia - que dans les opérations menées par les sociétés paramunicipales dans le VieuxMontréal depuis plus de vingt ans.

Le principe d'un tel partenariat est d'utiliser le financement public comme levier du développement privé, que ce soit en aménageant ou en réaménageant des espaces publics, afin de créer des lieux attrayants pour les promoteurs, ou encore en s'associant à des projets privés de développement qui, autrement, ne pourraient être réalisés.
De ce point de vue, le Quartier international constitue un site de choix, puisqu'il offre $48500 \mathrm{~m}^{2}$ au sol d'espaces à développer, en plein Centre des affaires. Le recouvrement de plusieurs tronçons de l'autoroute Ville-Marie offre une possibilité de développement et d'aménagement supplémentaires de près de $3200 \mathrm{~m}^{2}$ au sol (sans compter la surface du Palais des Congrès) et le secteur nord-ouest du Vieux-Montréal, adjacent au quartier, recèle $74300 \mathrm{~m}^{2}$ d'immeubles de grande valeur patrimoniale à rénover.

\section{LES PARTENAIRES ET LEUR RÔLE}

La Caisse de dépôt et placement du Québec a profité, en 1997, du projet d'agrandissement du Palais des Congrès pour relancer le projet de redéveloppement d'ensemble de la «Cité internationale », quelque peu laissé de côté. La Caisse estime que sa participation à un tel projet d'aménagement, susceptible de générer d'importants investissements à Montréal, répond parfaitement aux deux volets de sa mission : favoriser le rendement des sommes qui lui sont confiées et contribuer au dynamisme économique du Québec et de sa métropole. L'action de la Caisse de dépôt et placement du Québec a permis de confirmer le choix du site actuel du Palais des Congrès pour son agrandissement et de renforcer ainsi le rayonnement touristique du Palais des Congrès dans le Vieux-Montréal et le Quartier chinois.

La Ville de Montréal a été en quelque sorte l'initiatrice du concept même de «Cité internationale » : dès 1986, les grands partenaires socio-économiques, réunis à l'occasion du Sommet économique de Montréal, reconnaissaient les activités internationales comme un créneau à développer pour « redynamiser »la métropole. En 1989, la Ville lançait un concours international de design concernant l'aménagement de la Cité internationale. Les concurrents ont alors conclu à la nécessité de rendre convivial et accueillant pour les piétons un secteur qui avait jusqu'alors plutôt tendance à les rebuter ; ils ont proposé, notamment, de couvrir l'autoroute Ville- Marie, d'y aménager de nouveaux espaces publics, de réaménager les places existantes, de planter des arbres le long des rues principales, d'élargir les trottoirs et d'implanter des activités commerciales au rezde-chaussée des édifices, toutes des notions que l'on retrouve dans le projet actuel.

Au cours des années suivantes, la Ville réaffirmait son objectif de développer le caractère international de Montréal dans le secteur situé entre le Centre des affaires et le Vieux-Montréal et y facilitait l'implantation du Centre de commerce mondial et du siège de l'Organisation de l'aviation civile internationale (OACI). $\mathrm{La}$ participation de la Ville au projet actuel se situe donc en parfaite continuité avec ses actions depuis plus d'une décennie. 
Le troisième partenaire au projet est l'Association des riverains du Quartier international de Montréal (ARQIM), qui regroupe une vingtaine de riverains désireux de participer au redéveloppement de leur quartier, à la qualité de son environnement et à sa vitalité à long terme. Les membres de l'ARQIM assurent une part du financement privé du projet, par le biais d'une taxe d'amélioration locale. On y trouve des représentants de la grande entreprise québécoise, tels Power Corporation et Québécor, et du secteur immobilier, tels le Groupe Immobilier Caisse, MagilLaurentienne et Westcliff, ainsi que le Palais des Congrès de Montréal.

Les gouvernements du Québec et du Canada apportent une contribution financière déterminante au succès du projet. Par ailleurs, de grandes entreprises québécoises commanditent le projet d'ensemble et plusieurs sous-projets - comme la mise en lumière du secteur - contribuent à faire du quartier une vitrine des savoir-faire techniques québécois.

On peut aussi considérer les futurs riverains des espaces réaménagés du Quartier international - organisations internationales, hôtels, services financiers, consultants, etc. - comme des partenaires au projet. Parmi eux, la Caisse de dépôt et placement du Québec érigera, d'ici 2002, son nouveau bureau d'affaires montréalais à l'ouest de la place du Palais.

L'édifice, en forme de L renversé, est un bâtiment-pont qui reliera les deux îlots de part et d'autre de la rue Saint-Alexandre. La base du L comptera treize étages et la partie allongée en aura dix. Une veine vitrée liera les deux axes du L, ce qui favorisera la diffusion de la lumière naturelle dans une grande partie de l'édifice et permettra de recourir à des équipements bioclimatiques novateurs. Quelque 1200 personnes travailleront dans l'édifice qui regroupera toutes les activités de la Caisse : investissements immobiliers, placements privés, investissements sur les marchés.

Enfin, de nouveaux partenariats restent à développer en matière de promotion internationale pour optimiser les retombées du projet : on peut penser ici à Montréal International et au Palais des Congrès.

\section{UN ATTRAIT TOURISTIQUE EN SOI}

Au nombre des grands objectifs affichés dans le Programme particulier d'urbanisme du Quartier international (1999), se trouve celui «d'accroître les retombées déjà importantes du tourisme étranger (plus de 900 millions de dollars par année), des congrès (près de 200 millions de dollars par année) et des activités internationales à Montréal (valeur ajoutée nette de quelque 180 millions de dollars par année) ».

En ce qui concerne plus particulièrement les retombées touristiques, on peut affirmer que le Quartier international vise d'abord à devenir « un attrait touristique en soi », digne d'attirer les visiteurs étrangers par ses qualités propres.

Trois éléments interdépendants contribueront à l'atteinte de cet objectif :

1. les aménagements publics qui constituent l'essentiel du projet seront modernes, originaux et de grande qualité ;

2. ils contribueront à créer de nouvelles adresses de prestige qui pourront susciter des développements immobiliers d'une qualité architecturale et urbaine correspondante ;

3. ils feront des espaces publics du Quartier international une vitrine des ressources et des savoir-faire québécois.

Quant à la qualité des aménagements, notons d'abord que le projet conçu par le consortium Daoust Lestage-Provencher Roy \& Associés prend en compte tous les éléments de composition urbaine (structure paysagère, mise en lumière, mobilier urbain, art public). De plus, il privilégie l'utilisation des techniques de pointe (l'éclairage, l'animation des pièces d'eau, l'affichage électronique), la qualité du design (des aménagements et du mobilier urbain), la contribution de l'art public à l'animation des lieux et le recours aux matériaux et aux savoir-faire québécois.

Le réaménagement d'espaces publics existants (square Victoria) et l'aménagement de nouveaux espaces publics (place du Palais), l'élargissement des emprises de trottoirs, l'aménagement de corridors piétonniers protégés, la mise en lumière et la plantation d'arbres auront notamment pour effet de rendre le secteur plus accueillant et plus sûr, en augmentant de
$40 \%$ les surfaces piétonnières dans le secteur et en y créant des « sites propices à l'implantation de nouveaux riverains ». Il est clair que les mêmes critères de qualité, de modernité et d'originalité privilégiés dans l'aménagement des espaces publics devront s'appliquer au développement immobilier du secteur, de façon à donner au Quartier international un caractère distinctif.

La vitrine des ressources et des savoir-faire québécois, réalisée notamment avec la contribution des grandes sociétés québécoises de services publics, marquera les aménagements, la mise en lumière, l'intégration de l'art public et le mobilier urbain dans tout le quartier. Mais elle se cristallisera tout particulièrement dans les nouveaux liens piétonniers, composantes du réseau piétonnier protégé, lui-même une caractéristique originale et reconnue internationalement de l'aménagement urbain montréalais. Ces nouveaux liens, construits «à fleur de sol », seront en relation constante à la fois avec les infrastructures et les activités souterraines environnantes (autoroute, stationnement souterrain, métro, canalisations, évocation des techniques de sous-œuvre) et avec la surface (éclairage naturel, vue de l'extérieur et sur l'extérieur, apport d'air frais, continuité entre les espaces publics extérieurs et intérieurs).

L'aménagement de ces nouveaux liens protégés sera axé sur l'émergence historique, en jetant des ponts entre espaces, édifices et quartiers anciens et modernes, ainsi que sur l'intégration du développement souterrain et de surface, en donnant accès à des édifices qui offrent une activité commerciale au rez-de-chaussée (Palais des Congrès agrandi, Centre de commerce mondial, nouvel édifice de la Caisse de dépôt).

Ajoutons qu' une seconde phase du projet, de même qualité que la première, touchera les abords du Palais des Congrès agrandi.

\section{UN LIEN ENTRE LES GRANDS PÔLES TOURISTIQUES}

Au-delà de ses attraits propres, le Quartier international contribuera au tourisme par les liens qu'il créera ou rétablira entre les principales destinations touristiques montréalaises : Vieux-Montréal et VieuxPort au sud, centre-ville et Quartier chi- 
nois au nord, réseau piétonnier protégé à l'est et à l'ouest.

Deux interventions importantes, prévues au projet, établiront les liens : le recouvrement de l'autoroute Ville-Marie permettra d'éliminer une source de bruit, de viabiliser plus de $10700 \mathrm{~m}^{2}$ de terrain (dont les travaux liés à l'agrandissement du Palais des Congrès) et de recréer la trame urbaine dans tout le secteur qui va du centre-ville au Vieux-Montréal, tandis que la création de nouveaux liens piétonniers protégés, le premier reliant le hall du Palais des Congrès agrandi au square Victoria réaménagé et le second reliant la rue Saint-Jacques à ce premier lien par le Centre de commerce mondial, permettra de « boucler la boucle » du réseau piétonnier protégé.

À ce sujet, rappelons que le réseau piétonnier protégé de Montréal est l'un des plus importants au monde avec ses $30 \mathrm{~km}$ de couloirs donnant accès au transport public, à des centaines de commerces, de services, d'édifices à bureaux et de logements. En reliant entre eux les deux tronçons nordsud (axe Guy-Favreau / Complexe Desjardins / Place des Arts et axe Place Bonaventure / Gare Centrale / Place Ville-Marie / Centre Eaton), on pourra ainsi mettre en valeur, d'une façon nouvelle et systématique, ce réseau qui constitue, aux yeux des visiteurs étrangers, l'un des grands attraits touristiques de Montréal.

De plus, l'aménagement du nouveau lien est-ouest rapprochera le Palais des Congrès du bassin hôtelier du Centre des affaires, dans l'intérêt des congressistes qui séjournent à Montréal.

Par ailleurs, l'accessibilité, la circulation véhiculaire et piétonne et le stationnement sont autant d'aspects pris en compte dans le programme d'aménagement du quartier, afin d'y permettre une circulation sans entrave. Finalement, dans la seconde phase du projet, la Ville procédera au réaménagement de la partie sud de la rue McGill, qui assure le lien entre le Vieux-Port et le Quartier international.

\section{UN PROJET AUX EFFETS TONIFIANTS}

Un troisième apport du Quartier international au tourisme montréalais découle des effets de ce projet sur :
1. le développement des activités internationales à Montréal,

2. la bonification du projet d'agrandissement du Palais des Congrès,

3. la construction hôtelière dans le secteur.

Le développement de la vocation internationale du quartier s' appuie sur une forte interaction des activités d'affaires et des activités touristiques. En effet, l'accueil des visiteurs internationaux se traduit par des infrastructures et des services conjoints localisés à la jonction des pôles d'affaires et des pôles touristiques.

L'aménagement du Quartier international vise en tout premier lieu à consolider la vocation du territoire en y facilitant 1 'implantation d'autres grandes organisations internationales, notamment des secteurs d'excellence montréalais : aviation, environnement, finance, droit international, etc. Mais on peut aussi prévoir l'arrivée d'entreprises desservant ces organisations et leur clientèle : hôtels, institutions financières, consultants, etc. La croissance de telles activités suppose un flux important de visiteurs étrangers.

S'il est juste de dire que c'est le projet d'agrandissement du Palais des Congrès qui a fourni l'occasion de relancer le projet du Quartier international, il est tout aussi vrai que l'élaboration de ce dernier a incité l'équipe du Palais des Congrès à bonifier son projet de façon à le rendre plus accueillant, plus convivial et mieux intégré à l'environnement urbain : à titre d'exem-
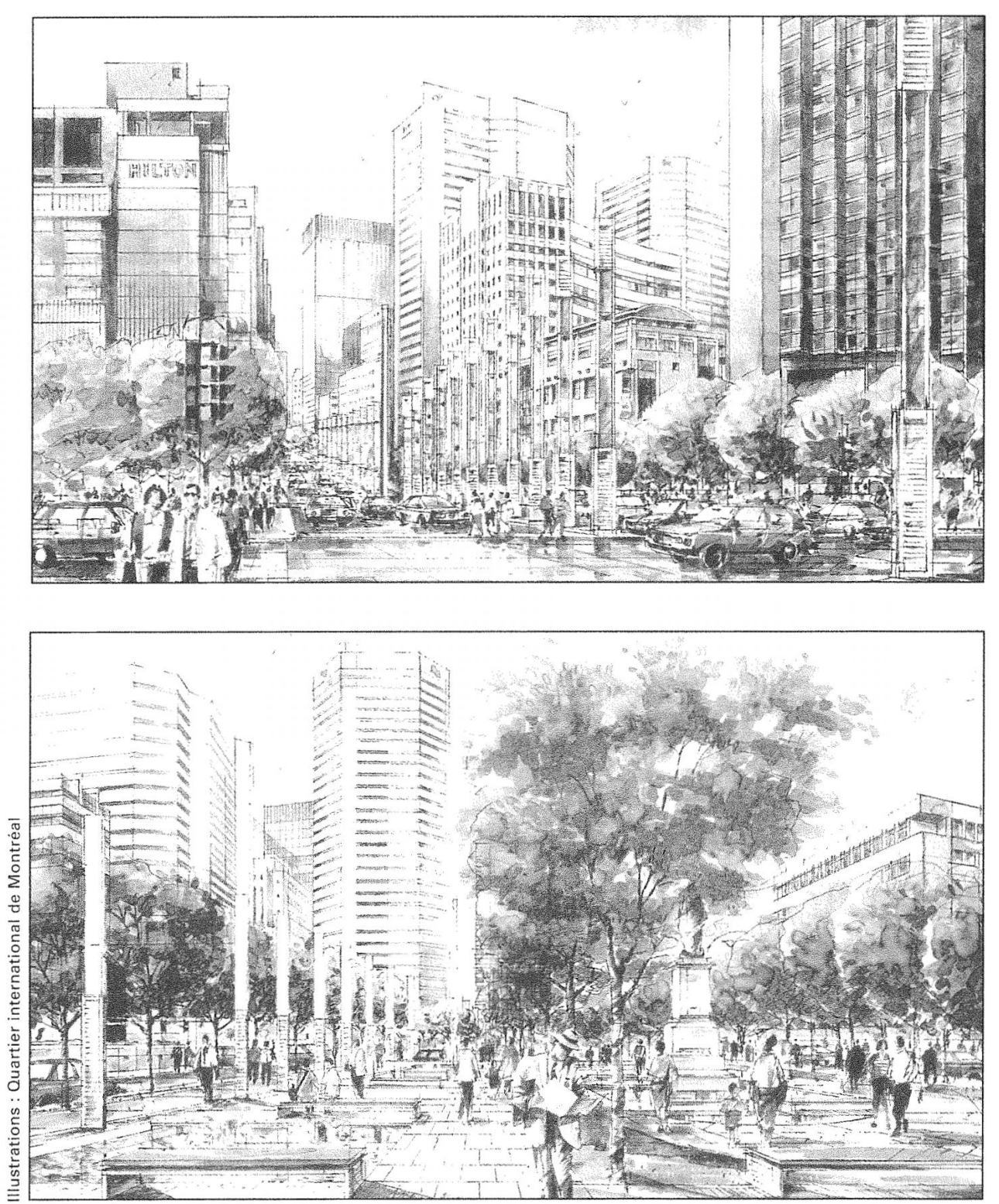


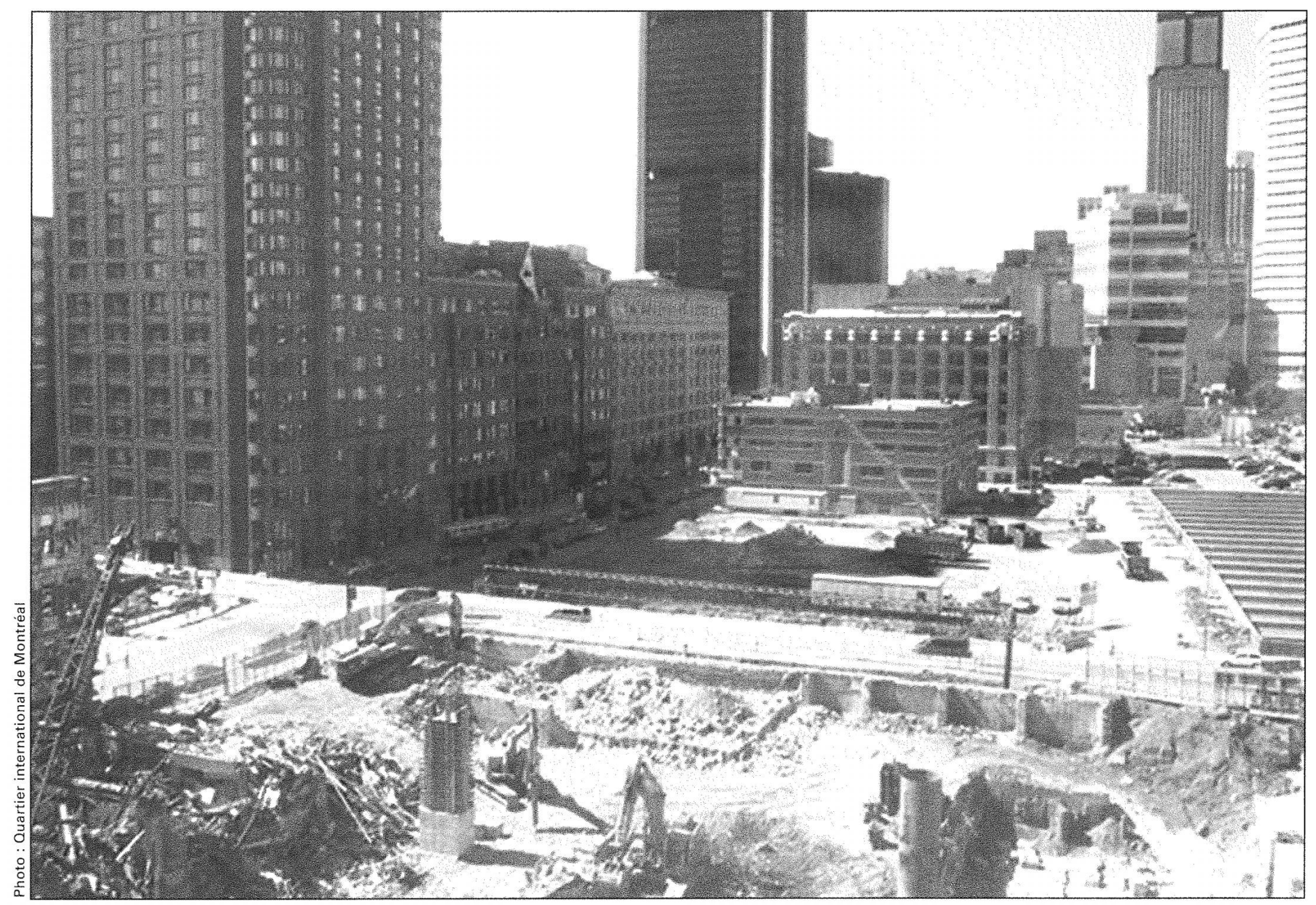

Le chantier de construction

ple, la portion de l'autoroute Ville-Marie située sous le Palais des Congrès, zone particulièrement bruyante aujourd'hui, sera recouverte pour accueillir un rez-de-chaussée commercial, largement ouvert sur la rue et traversé d'est en ouest par un vaste couloir piétonnier menant du métro Placed'Armes à la nouvelle entrée monumentale, rue de Bleury, et à la place du Palais.

Ainsi amélioré et ouvert à son environnement, le Palais incitera désormais ses congressistes à s'aventurer dans le VieuxMontréal, le Vieux-Port, le Centre de commerce mondial, le Quartier international et le centre-ville, et - qui sait ? - à revenir en touristes à Montréal.

Enfin, le développement du Quartier international aura, avec l'agrandissement du Palais des Congrès et la croissance de la Cité du multimédia, un effet moteur sur la construction hôtelière, notamment dans le Vieux-Montréal. En effet, des projections effectuées en 1999 par la firme Horwath démontrent que le marché hôtelier du Vieux-Montréal et de sa zone d'influence pourrait supporter, d'ici 2008, l'ajout de 1200 à 1500 chambres. D'où 1'opportunité de développer des produits hôteliers distinctifs : hôtels conventionnels de première classe, comptant 350 à 500 chambres, pour répondre aux besoins de la clientèle du Palais des Congrès; hôtels de première classe ou intermédiaires, comptant 50 à 150 chambres et, surtout, auberges de première classe ou intermédiaires, comportant moins de 50 chambres et reflétant, par leur caractère intimiste et personnalisé, le cachet du quartier historique.

\section{UN EFFET MULTIPLICATEUR}

Les partenaires du Quartier international répondent avec un enthousiasme communicatif au projet de développer un secteur prestigieux et convivial du centre-ville, destiné à affirmer l'identité internationale de Montréal. Au-delà du réaménagement urbain et du développement immobilier du centre-ville, leur démarche commune s'appuie sur le pari audacieux de l'effet multiplicateur des activités internationales et touristiques de Montréal sur l'économie nationale. Déjà, les investisseurs semblent leur donner raison puisque le secteur compris entre le Palais des Congrès et la place Bonaventure est devenu le plus vaste chantier au Canada.

Clément Demers, architecte, urbaniste et gestionnaire de projet, est le directeur général adjoint du Quartier international de Montréal ; Bernard Lamothe est consultant en communication et Louis-François Monet, urbaniste, est directeur général adjoint du Quartier international de Montréal. 